\title{
Measurement Accuracy of Artificial Intelligence in the Form of Convolutional Neural Networks for Cervical Lordosis on Lateral Radiographs
}

Takahito Fujimori ( $\nabla$ takahito-f@hotmail.co.jp )

Osaka University

Yuki Suzuki

Osaka University

Kosuke Kita

Osaka University

Yuya Kanie

Osaka University

Shota Takenaka

Osaka University

Takashi Kaito

Osaka University

Yuichiro Ukon

Osaka University

Tadashi Watabe

Osaka University

Shoji Kido

Osaka University

\section{Seiji Okada}

Osaka University

\section{Research Article}

Keywords: alignment, artificial intelligence, automated measurement, cervical x-rays, convolutional neural networks, deep learning

Posted Date: January 31st, 2022

DOI: https://doi.org/10.21203/rs.3.rs-1224098/v1

License: (c) (1) This work is licensed under a Creative Commons Attribution 4.0 International License.

Read Full License 


\section{Abstract}

Although cervical alignment is important for evaluating spine disorders, manual measurement is timeconsuming and burdensome. We aimed to validate the usefulness of artificial intelligence (Al) in the form of convolutional neural networks for automated measurement of lordosis on lateral cervical $x$-rays. We included 4546 cervical x-rays from 1674 patients. For all x-rays, a well-experienced spine surgeon labeled the caudal endplates of $\mathrm{C} 2$ and $\mathrm{C} 7$, the data for which were used as ground truth. The accuracy of $\mathrm{Al}$ measurements was tested by 5 -fold cross-validation and by comparison with measurements obtained by 2 surgeons. The mean absolute error (MAE) of the Al model in 5 -fold cross-validation was $3.6^{\circ} \pm 5.5^{\circ}$ at the $\mathrm{C} 2-\mathrm{C} 7$ angle, and the model took 206 seconds to measure $4546 \mathrm{x}$-rays. The MAE for measurement of 416 radiographs of 168 randomly selected patients was $3.3^{\circ} \pm 3.8^{\circ}$ for the Al model, $3.9^{\circ} \pm 3.4^{\circ}$ for Surgeon 1 , and $3.8^{\circ} \pm 4.7^{\circ}$ for Surgeon 2 . Thus, the Al model had a significantly smaller error than Surgeon 1, and its error was not significantly different from that of Surgeon 2.

In conclusion, Al can assist in routine medical care and can be helpful in research that measures large numbers of images.

\section{Introduction}

Cervical alignment, an important clinical parameter in spine disorders, is associated with deformity, myelopathy, adjacent-segment disease, horizontal gaze, and health-related quality of life. ${ }^{1,2}$ Measuring cervical alignment in multiple positions is important in evaluating pathology and planning surgery.

Historically, such measurements have been obtained by using a protractor on radiographs. In recent years, digital viewer measurements became more common, ${ }^{3}$ but surgeons generally still had to obtain measurements manually. Obtaining the necessary measurements for many parameters before and after surgery for a large number of patients requires a great deal of labor. Artificial intelligence (Al) models using convolutional neural networks (CNNs), a type of machine learning, have excellent capabilities for image recognition. ${ }^{4-6}$ Because they require relatively less preprocessing than other algorithms, and because they automatically learn to optimize filters, whereas traditional algorithms do so manually, ${ }^{7,8}$ they may reduce the labor involved in measurement.

A recent study ${ }^{9}$ of CNNs showed that the standard error for determining lumbar lordosis in scoliosis was $11.5^{\circ}$. Other studies have reported a mean absolute error (MAE) ranging from $4.3^{\circ}$ to $8.1^{\circ}$ when $\mathrm{Al}$ is used to assess lumbar lordosis. ${ }^{10,11}$ There is room for improvement in the measurement accuracy of Al models that use CNNs. We thus conducted a study with the aim of determining the usefulness of CNN-based $\mathrm{Al}$ in automated measurement of the $\mathrm{C} 2-\mathrm{C} 7$ angle on cervical $\mathrm{x}$-rays.

\section{Methods}


This study was approved by our institution's review board (Osaka University Hospital Ethics Review Committee. No.20416) and written informed consent was waived because of the retrospective design. The study was performed in accordance with approved guidelines and in compliance with the principles of the Declaration of Helsinki.

\section{Study Participants}

Study participants were selected in two ways: First, we searched the list of patients who underwent cervical spine surgery in our spine clinic at some point between May 2012 and December 2020, and second, we searched the list in the medical information system for patients who had cervical x-rays obtained at our hospital at some point between April 2019 and April 2021. From the two lists, we chose to include 1674 patients with a total of $4546 \mathrm{x}$-rays, excluding patients who underwent radiography more than once. To validate the capability of Al in real-world clinical practice, we did not exclude any patients who had deformities or who underwent spinal instrumentation, and all patients from the two lists were included in our study. All x-rays were measured on the lateral view and included flexion, extension, and the neutral position. X-rays were downloaded in DICOM (Digital Imaging and Communications in Medicine) file format and converted to PNG (Portable Network Graphic) file format.

\section{Method of Radiographic Measurement}

We used the Cobb method to measure the $\mathrm{C} 2-\mathrm{C} 7$ angle because it is simple and most commonly used. ${ }^{1,12}$ We labeled the anterior and posterior endpoints of the $\mathrm{C} 2$ inferior endplate as anatomic landmarks in a digital viewer to draw a straight line along the $\mathrm{C} 2$ inferior endplate, and we used the same method for the $\mathrm{C} 7$ vertebra (Figure 1). If the $\mathrm{C} 7$ vertebral body was obscured by the shoulder girdle and difficult to see, we used the $\mathrm{C} 6$ vertebral endplate as a reference for the $\mathrm{C} 7$ vertebral endplate. We used a publicly available image annotation software labelme (https://github.com/wkentaro/labelme) for this manual measurement process.

We labeled the $\mathrm{C} 2$ slope and the $\mathrm{C} 7$ slope, which are the angles that the $\mathrm{C} 2$ lower endplate and the $\mathrm{C} 7$ lower endplates make with the horizontal line, with clockwise being positive in both cases. The angle obtained by subtracting the $\mathrm{C} 2$ slope from the $\mathrm{C} 7$ slope is the $\mathrm{C} 2-\mathrm{C} 7$ angle, with a negative angle indicating lordosis and a positive angle indicating kyphosis.

\section{Artificial Intelligence Model}

The Al model detected four anatomic landmarks: the anterior and posterior endpoints of the $\mathrm{C} 2$ and $\mathrm{C} 7$ inferior endplates. This anatomic landmark localization was performed by using CNNs to produce a heat map and then extracting the coordinates with the maximum value from the heat map of each landmark ${ }^{13}$ (Figure 2).

For the CNNs to output heat maps, we used the DeepLabV3 segmentation architecture, ${ }^{14}$ with the EfficientNet-B4 scaling method ${ }^{15}$ as a backbone. DeepLabV3 is a segmentation architecture that uses atrous convolution to enlarge the field of view of the network and Efficient Net-B4 is a classification 
model that was designed to balance the model size and the model accuracy. CNNs and angle measurements were implemented using Python version 3.9.5 (an interface) and PyTorch version 1.8.1 (an open-source machine learning framework). Our model was built using Segmentation Models Pytorch (https://github.com/qubvel/segmentation_models.pytorch), which is a publicly available package of Python and the backbone (EfficientNet-B4) was pretrained with ImageNet. The training of CNN was performed using Adam optimizer with initial learning rate of 0.0001 using the root mean square as the loss function until the loss of the validation data extracted from the training data started to drop (i.e., just before overfitting).

The value on the heat map for each landmark was used as the confidence score, and the smallest of the four values was used as the confidence score for that $x$-ray. We used confidence scores for later analysis.

\section{Creation of Ground Truth Data and Validation of Accuracy}

A spine surgeon with 18 years' experience labeled the C2 and C7 endplates on all $4546 \mathrm{x}$-rays, and we used this as the ground truth. In machine learning, ground truth is labeled data that are considered to be the correct values. Ground truth data are divided into training data and test data. We examined measurement accuracy using two techniques.

The first technique involved the error of the Al algorithm's measurements relative to the ground truth, calculated by 5 -fold cross-validation. We randomly divided all ground truth data into five groups: four groups were training data, and one group was test data. The algorithm learned the training data of the four groups and measured the test data of the remaining one group. We then calculated the absolute error of the algorithm's measurements and the ground truth measurements on the test data (Figure 3). This process was repeated five times, changing the training and test data groups so that all data were tested. Finally, the average of these absolute errors obtained from five processes represents the accuracy of the algorithm's measurements. We did this five-grouping on a case-by-case basis, not on the basis of each x-ray; the CNNs did not learn from x-rays of the same patient in different positions. We performed validation on a workstation with two NVIDIA computers with GeForce RTX 3090 graphics-processing units, and the CNNs and angle measurements were implemented using Python (an interface) and PyTorch (an open-source machine learning framework). The training of each CNN was performed until the accuracy of the validation data extracted from the training data dropped (i.e., just before overfitting).

The second technique involved comparing the accuracy of the algorithm's measurements with that of surgeons. Surgeon 1, with 11 years' experience, and Surgeon 2, with 7 years' experience, were both spine surgeons. From 1674 patients, we randomly selected 168 patients ( 57 men and 111 women) with a total of $416 \mathrm{x}$-rays, and each surgeon measured these according to the Cobb method described in the section "Method of Radiographic Measurement." The surgeon who created the ground truth also measured again more than 1 month later, recording data at that point as Surgeon 3. The CNNs were trained on 1506 patients (4130 x-rays), excluding the 168 test patients, and measured on 168 patients (416 x-rays). We compared the error for the Al algorithm with the error for Surgeon 1, for Surgeon 2, and for Surgeon 3.

\section{Repeatability and Measurement Time}


For the Al algorithm versus the surgeons, we compared the repeatability of measurements and the time needed to obtain measurements. The intraclass correlation coefficient of the two measurements of the ground truth surgeon (Surgeon 3) was used as surgeon repeatability. Surgeon 3 recorded the time needed to measure $10 \mathrm{x}$-rays and calculated the average value per $\mathrm{x}$-ray; the Al algorithm recorded the time to measure all $4546 \mathrm{x}$-rays and calculated the average value per $\mathrm{x}$-ray.

\section{Setting the Confidence Score}

We set the confidence score to measure the level of confidence in the measurements of the Al algorithm. The confidence score is expressed as a value between 0 and 1 , where 0 indicates no confidence and 1 indicates confidence. Excluding x-rays with a low confidence score was expected to reduce the absolute error. By varying the confidence score as a threshold, we examined the relationship between the number of excluded x-rays and the absolute error.

\section{Relationship Between the Absolute Error of Artificial Intelligence and Background Data on Participants}

We performed a multivariate analysis with absolute error as the objective variable and with age, sex, whether the patient had undergone surgery, and cervical spine position (flexion, neutral, and extension) as explanatory variables. The absolute errors were compared between the group of patients who had undergone surgery and the group of those who had not.

\section{Statistical Analysis}

We used the $t$ test to compare absolute errors between surgeons against such errors by the Al system and to compare errors regarding patients who underwent surgery and those who did not. Stepwise multiple regression analysis was performed with the absolute error at the $\mathrm{C} 2-\mathrm{C} 7$ angle as the dependent variable and the patients' demographic data as the independent variable. $P$ values $<0.05$ (two-sided) were considered statistically significant. Statistical analysis was performed using the SPSS Statistics software (version 20; IBM, Armonk, NY, USA).

\section{Results}

\section{Demographic Data}

A total of 1674 patients with 4546 x-rays were included in our study: 707 males and 967 females (Table 1). The mean age was $61 \pm 19$ years (range, $2-96$ years). 
Table 1

Demographic Data of Study Participants

\begin{tabular}{|lll|}
\hline Variable & Patients & Cervical X-rays \\
\hline Number & 1674 & 4546 \\
\hline Men & 707 & 2060 \\
\hline Women & 967 & 2486 \\
\hline Mean age (years) & $61 \pm 18$ & N/A \\
\hline Minimum age (years) & 2 & N/A \\
\hline Maximum age (years) & 96 & N/A \\
\hline Patients underwent surgery & 280 & 877 \\
\hline Patients did not undergo surgery & 1394 & 3669 \\
\hline N/A, not applicable. & & \\
\hline
\end{tabular}

Using the ground truth as a basis, we found the measurements to be $-9.5^{\circ} \pm 15.6^{\circ}$ in the neutral position, $13.9^{\circ} \pm 15.8^{\circ}$ in flexion, and $-25.0^{\circ} \pm 18.4^{\circ}$ in extension (Table 2). Surgical cases involved 280 participants $(17 \%)$ with a total of $877 \mathrm{x}$-rays.

Table 2

X-ray Measurements at Each Position Based on the Ground Truth

\begin{tabular}{|lllll|}
\hline Position & Number of X-rays & $\begin{array}{l}\text { C2-C7 Angle } \\
\text { (degrees) }\end{array}$ & $\begin{array}{l}\text { C2 Slope } \\
\text { (degrees) }\end{array}$ & $\begin{array}{l}\text { C7 Slope } \\
\text { (degrees) }\end{array}$ \\
\hline Flexion & 1460 & $13.9 \pm 15.8$ & $-42.9 \pm 17.7$ & $-29.9 \pm 11.6$ \\
\hline Neutral & 1643 & $-9.5 \pm 15.6$ & $-17.0 \pm 13.2$ & $-26.7 \pm 10.6$ \\
\hline Extension & 1443 & $-25.0 \pm 18.4$ & $0.2 \pm 16.5$ & $-24.8 \pm 10.6$ \\
\hline
\end{tabular}

\section{Absolute Error of Artificial Intelligence Relative to Ground Truth}

The MAE of the CNNs in all 1674 patients (with a total of 4546 x-rays) was $3.6^{\circ} \pm 5.5^{\circ}$ for the $\mathrm{C} 2-\mathrm{C} 7$ angle, $1.8^{\circ} \pm 2.9^{\circ}$ for the $\mathrm{C} 2$ slope, and $3.0^{\circ} \pm 4.7^{\circ}$ for the $\mathrm{C} 7$ slope. The median absolute error was $2.4^{\circ}$ for the $\mathrm{C} 2-\mathrm{C} 7$ angle, $1.3^{\circ}$ for the $\mathrm{C} 2$ slope, and $1.9^{\circ}$ for the $\mathrm{C} 7$ slope. The maximum absolute error was $127.9^{\circ}$ for the $\mathrm{C} 2-\mathrm{C} 7$ angle, $79.3^{\circ}$ for the $\mathrm{C} 2$ slope, and $85.7^{\circ}$ for the $\mathrm{C} 7$ slope.

\section{Relationship Between Confidence Score and Absolute Error}

The mean confidence score was $0.95 \pm 0.05$ for the $\mathrm{C} 2$ slope and $0.88 \pm 0.15$ for the $\mathrm{C} 7$ slope (Figure 4A). When the threshold was set to $0.6,294$ x-rays (6.5\%) were excluded, and the MAE in the C2-C7 angle 
dropped to $2.9^{\circ}$, the median to $2.25^{\circ}$, and the maximum error to $23.5^{\circ}$. Similarly, when the threshold was set at $0.9,1803$ x-rays $(39.7 \%)$ were excluded, and the MAE in the $\mathrm{C} 2-\mathrm{C} 7$ angle dropped to $2.4^{\circ}$, the median to $1.99^{\circ}$, and the maximum error to $16.6^{\circ}$ (Figures $4 \mathrm{~B}, 4 \mathrm{C}$ ).

Comparison of Absolute Error at the C2-C7 Angle for Randomly Selected Participants Relative to Ground Truth Between Artificial Intelligence and Surgeons

\section{Artificial Intelligence}

In the group of randomly selected patients (comprising 168 cases with 416 total x-rays), the MAE of the Al algorithm was $3.3^{\circ} \pm 2.8^{\circ}$, the median absolute error was $2.3^{\circ}$, and the maximum absolute error was $31.9^{\circ}$ (Figure 5 and Table 3).

Table 3

Comparison of Errors Between Al and Surgeons for Randomly Selected Cases*

\begin{tabular}{|lllll|}
\hline Value & Al & Surgeon 1 & Surgeon 2 & Surgeon 3 \\
\hline Mean absolute error (degrees) & & & & \\
\hline C2-C7 angle & 3.29 & 3.91 & 3.78 & 2.48 \\
\hline C2 slope & 1.71 & 2.35 & 2.13 & 1.42 \\
\hline C7 slope & 2.79 & 3.12 & 3.33 & 2.07 \\
\hline Median absolute error (degrees) & & & & \\
\hline C2-C7 angle & 2.28 & 3.03 & 2.94 & 1.70 \\
\hline C2 slope & 1.29 & 1.94 & 1.69 & 1.11 \\
\hline C7 slope & 1.70 & 2.16 & 2.22 & 1.52 \\
\hline Maximum absolute error (degrees) & & & & \\
\hline C2-C7 angle & 31.9 & 22.1 & 74.1 & 18.4 \\
\hline C2 slope & 9.69 & 17.8 & 9.70 & 7.07 \\
\hline C7 slope & 35.4 & 22.5 & 73.5 & 18.0 \\
\hline Standard deviation (degrees) & & & & \\
\hline C2-C7 angle & 3.82 & 3.38 & 4.72 & 2.38 \\
\hline C2 slope & 1.49 & 2.09 & 1.81 & 1.16 \\
\hline C7 slope & 3.92 & 2.90 & 4.71 & 2.07 \\
\hline *168 cases with a total of 416 x-rays. & & & \\
\hline Al, artificial intelligence. & & & & \\
\hline
\end{tabular}




\section{Surgeon 1}

For Surgeon 1 , the MAE was $3.9^{\circ} \pm 3.4^{\circ}$, the median absolute error was $3.0^{\circ}$, and the maximum absolute error was $22.1^{\circ}$.

\section{Surgeon 2}

For surgeon 2 , the MAE was $3.8^{\circ} \pm 4.7^{\circ}$, the median absolute error was $2.9^{\circ}$, and the maximum absolute error was $74.1^{\circ}$.

\section{Surgeon 3 (Ground Truth Surgeon)}

For Surgeon 3 (the ground truth surgeon), the MAE was $2.5^{\circ} \pm 2.4^{\circ}$, the median absolute error was $1.7^{\circ}$, and the maximum absolute error was $18.4^{\circ}$.

\section{Statistical Results}

The Al algorithm had a significantly smaller absolute error than Surgeon $1 \mathrm{did}(P=0.013)$. There was no significant difference in error between the algorithm and Surgeon $2(P=0.1)$, but the algorithm had a significantly larger error than Surgeon 3 did $(P=0.001)$.

\section{Repeatability and Measurement Time}

The intraclass correlation coefficient for the Al algorithm was 1.0, whereas it was 0.990 (95\% confidence interval: 0.992-0.988) for Surgeon 3.

Surgeon 3 took 196 seconds to measure 10 x-rays, at an average speed of 19.6 seconds per $x$-ray. The Al algorithm, however, took 206 seconds to measure 4546 x-rays, at an average speed of 0.045 seconds per x-ray.

Relationship Between Absolute Error at the C2-C7 Angle for Artificial Intelligence and Background Data on Participants

A stepwise multivariate analysis was performed regarding age, sex, whether the patient had undergone surgery, and radiographic posture (flexion, neutral position, extension) as independent variables. Being of younger age, being male, and having undergone surgery were related to a larger error rate (Table 4). The MAE for participants who underwent surgery $\left(4.2^{\circ} \pm 6.3^{\circ}\right)$ was significantly larger than for those who did not undergo surgery $\left(3.4^{\circ} \pm 5.3^{\circ} ; P<0.001 ;\right.$ Table 5$)$. 
Table 4

Stepwise Multiple Regression Analysis of Absolute Error at the C2-C7 Angle as the Dependent Variable

\begin{tabular}{|llllll|}
\hline Independent Variables & \multicolumn{3}{c}{ Covariates } & & \\
\cline { 2 - 6 } & B & SE & Beta & t Test & P Value \\
\hline Age & -0.036 & 0.005 & -0.108 & -7.145 & $<0.000$ \\
\hline Undergoing surgery & 0.866 & 0.212 & 0.062 & 4.086 & $<0.000$ \\
\hline Sex (male) & 0.373 & 0.169 & 0.034 & 2.212 & 0.027 \\
\hline The square of the coefficient of multiple correlation $\left(\mathrm{R}^{2}\right)$ in this model $=0.016$. & \\
B, partial regression coefficient; & SE, standard error; beta, standardized partial regression coefficient. \\
\hline
\end{tabular}

Table 5

Comparison of the Errors Between Surgical and Nonsurgical Cases When Measured by Artificial Intelligence

\begin{tabular}{|lll|}
\hline Variable & Surgery Involved & No Surgery Involved \\
\hline Number of patients & 280 & 1394 \\
\hline Number of x-rays & 877 & 3669 \\
\hline Mean absolute error of C2-C7 angle \pm SD (degrees) & $4.2 \pm 6.3$ & $3.4 \pm 5.3$ \\
\hline SD, standard deviation. & & \\
\hline
\end{tabular}

\section{Participants with Absolute Error of $20^{\circ}$ or More at the C2- C7 Angle}

There were 61 participants with an absolute error of $\geq 20^{\circ}$ at the $\mathrm{C} 2-\mathrm{C} 7$ angle. The MAEs for these participants were $36.9^{\circ}$ for the $\mathrm{C} 2-\mathrm{C} 7$ angle, $10.6^{\circ}$ for the $\mathrm{C} 2$ slope, and $28.5^{\circ}$ for the $\mathrm{C} 7$ slope. The mean confidence score was 0.34 . Most of the errors were due to mistakes in measurement of the $\mathrm{C} 7$ slope, which was caused by misidentifying a different vertebral body as $\mathrm{C7}$. The error was huge when a line was drawn connecting the anterior edge of the inferior endplate of one vertebra with the posterior edge of the inferior endplate of a different vertebra. The most common reasons for vertebral body misidentification were the presence of severe spine deformity $(n=15 ; 25 \%)$, no visible C7 $(n=14 ; 23 \%)$, the presence of fused vertebrae after anterior fusion $(n=12 ; 20 \%)$, the use of posterior instrumented fusion $(n=10 ; 16 \%)$, and patients being in their infancy $(n=8 ; 13 \%)$.

\section{Discussion}

We created an Al model for 1674 participants (with a total of 4546 cervical x-rays), with no participants being excluded. CNNs measured the $\mathrm{C} 2-\mathrm{C} 7$ angle with a MAE of $3.6^{\circ}$ and a median absolute error of $2.4^{\circ}$. The Al algorithm was almost as good as surgeons regarding accuracy, and it was much better regarding 
the amount of time required for measurement and regarding reproducibility. Accuracy was improved by adjusting the confidence score.

To the best of our knowledge, ours is the first study to use Al to measure cervical sagittal alignment on $x$ rays. There have been some earlier reports, however, on automated measurement of the lumbar spine. Cho et al ${ }^{11}$ used Al to measure L1-S1 lumbar lordosis in 780 lumbar spine x-rays from 780 people, excluding those who had undergone surgery, successfully measuring lordosis in $84 \%$ of their study participants, with an MAE of $8.055^{\circ}$ and a median absolute error of $6.965^{\circ}$. Schwartz et al ${ }^{10}$ used Al to measure L1-S1 lumbar lordosis in 816 lumbar spine x-rays of 816 patients older than age 18 years, including $6.1 \%$ who underwent spinal instrumentation. The MAE was $4.3^{\circ}$, and the median absolute error was $2.2^{\circ}$. Korez et al ${ }^{16}$ measured spinopelvic parameters in 55 patients using $\mathrm{Al}$ and reported that the MAE ranged from $1.2^{\circ}$ to $5.5^{\circ}$.

In general, the cervical spine may be more challenging to measure than the lumbar spine because the shoulder girdle may hide $\mathrm{C} 7$. However, our results were better than for previous measurements of the lumbar spine. One reason for the smaller absolute error of $\mathrm{Al}$ in our study is that we had more training data; a second reason is that there were fewer processing steps. Previous researchers manually segmented all vertebrae, extracted the vertebrae, and then measured the angle. However, we could directly measure the angle by annotating only the vertebral vertices needed for the angle measurement. This reduced the number of processing steps and reduced the absolute error. The reduction in our annotation process also contributed to the increase in our training data.

One of the limitations of Al in our study was that the maximum absolute error was large. Because we excluded no participants, advanced deformities such as congenitally fused or malformed vertebrae were included. Some of the advanced deformities were difficult to measure, even for surgeons. It is challenging to deal with Al that is based on machine learning because the correct answer is not apparent for the tasks involved. In addition, advanced deformities are rare, so there is a lack of training data for them. When such rare deformities are included, it seems unavoidable that absolute errors will be large. Factors related to the absolute error in our study included being of younger age, having undergone surgery, and being male. This was thought to be the case because of the lack of training data in pediatric and surgical cases. The large absolute error in men may be because their shoulder girdle often hides the $\mathrm{C} 7$ vertebral body.

To fully automate measurement with Al, it is desirable to further increase the amount of training data for rare deformities. However, depending on the purpose of using Al for measurements, the current performance level may be sufficient. For example, if $x$-rays are obtained for a healthy person for a medical checkup and that the person has not undergone surgery, then the measurement can be done quite accurately. If surgeons use Al for clinical research, they can control the error by adjusting the confidence score. Surgeons take a long time to measure manually, and their work efficiency decreases over time because of fatigue. However, Al can take measurements quickly, and there is no such decrease in work efficiency. In addition, simply incorporating an Al measurement function into an existing image 
viewer to assist in measurement will greatly improve work efficiency. Surgeons do not need to measure manually but only need to check Al measurement lines. If surgeons determine that the measurement is incorrect, they can correct it. As Al learns more and more, it is expected to become more and more accurate. In conclusion, we have successfully developed an Al tool for rapid and accurate automated measurement of cervical $x$-rays. These tools have a high clinical application value.

\section{Declarations}

\section{Contributions}

Takahito Fujimori and Yuki Suzuki conceived the study. Takahito Fujimori, Yuki Suzuki, Kosuke Kita, and Yuya Kanie contributed to the data collection. Takahito Fujimori, and Yuki Suzuki contributed to the imaging data analysis. Takahito Fujimori wrote the initial draft. Yuki Suzuki, Shota Takenaka, and Takashi Kaito revised the draft. All authors reviewed the manuscript.

\section{Competing Interests}

The authors declare no competing interests.

\section{Acknowledgments}

This work was supported by JSPS KAKENHI grant number JP21K20966. Tetsuhisa Kitamura provided statistical advice for this article. Medical editor Katharine O'Moore-Klopf, ELS (East Setauket, NY, USA) provided professional English-language editing of this article.

\section{Data Availability}

The datasets generated during and/or analysed during the current study are available from the corresponding authors on reasonable request.

\section{References}

1. Scheer, J. K. et al. Cervical spine alignment, sagittal deformity, and clinical implications: a review. J Neurosurg Spine 19, 141-159, doi:10.3171/2013.4.SPINE12838 (2013).

2. Fujimori, T. et al. Long-term results of cervical myelopathy due to ossification of the posterior longitudinal ligament with an occupying ratio of $60 \%$ or more. Spine (Phila Pa 1976) $39,58-67$, doi:10.1097/BRS.0000000000000054 (2014).

3. Lafage, R. et al. Validation of a new computer-assisted tool to measure spino-pelvic parameters. Spine Journal 15, 2493-2502, doi:10.1016/j.spinee.2015.08.067 (2015).

4. McKinney, S. M. et al. International evaluation of an Al system for breast cancer screening. Nature 577, 89-94, doi:10.1038/s41586-019-1799-6 (2020). 
5. Guo, Y. M. et al. Deep learning for visual understanding: A review. Neurocomputing 187, 27-48, doi:10.1016/j.neucom.2015.09.116 (2016).

6. Miura, M. et al. Automated detection of cervical ossification of the posterior longitudinal ligament in plain lateral radiographs of the cervical spine using a convolutional neural network. Sci Rep 11, 12702, doi:10.1038/s41598-021-92160-9 (2021).

7. Zhang, J. H., Li, H. J., Lv, L. \& Zhang, Y. F. Computer-Aided Cobb Measurement Based on Automatic Detection of Vertebral Slopes Using Deep Neural Network. Int J Biomed Imaging 2017, doi:Artn 9083916 1155/2017/9083916 (2017).

8. Chartrand, G. et al. Deep Learning: A Primer for Radiologists. Radiographics 37, 2113-2131, doi:10.1148/rg.2017170077 (2017).

9. Galbusera, F. et al. Fully automated radiological analysis of spinal disorders and deformities: a deep learning approach. Eur Spine J 28, 951-960, doi:10.1007/s00586-019-05944-z (2019).

10. Schwartz, J. T. et al. Deep Learning Automates Measurement of Spinopelvic Parameters on Lateral Lumbar Radiographs. Spine (Phila Pa 1976) 46, E671-E678, doi:10.1097/BRS.0000000000003830 (2021).

11. Cho, B. H. et al. Automated Measurement of Lumbar Lordosis on Radiographs Using Machine Learning and Computer Vision. Global Spine J 10, 611-618, doi:10.1177/2192568219868190 (2020).

12. Janusz, P., Tyrakowski, M., Yu, H. \& Siemionow, K. Reliability of cervical lordosis measurement techniques on long-cassette radiographs. Eur Spine J 25, 3596-3601, doi:10.1007/s00586-0154345-8 (2016).

13. Cao, Z., Simon, T., Wei, S. E. \& Sheikh, Y. Realtime Multi-Person 2D Pose Estimation using Part Affinity Fields. Proc Cvpr leee, 1302-1310, doi:10.1109/Cvpr.2017.143 (2017).

14. Chen, L.-C., Papandreou, G., Schroff, F. \& Adam, H. Rethinking atrous convolution for semantic image segmentation. arXiv 2017. arXiv preprint arXiv:1706.05587(2019).

15. Tan, M. \& Le, Q. in International Conference on Machine Learning. 6105-6114 (PMLR).

16. Korez, R., Putzier, M. \& Vrtovec, T. A deep learning tool for fully automated measurements of sagittal spinopelvic balance from X-ray images: performance evaluation. Eur Spine J 29, 2295-2305, doi:10.1007/s00586-020-06406-7 (2020).

\section{Figures}




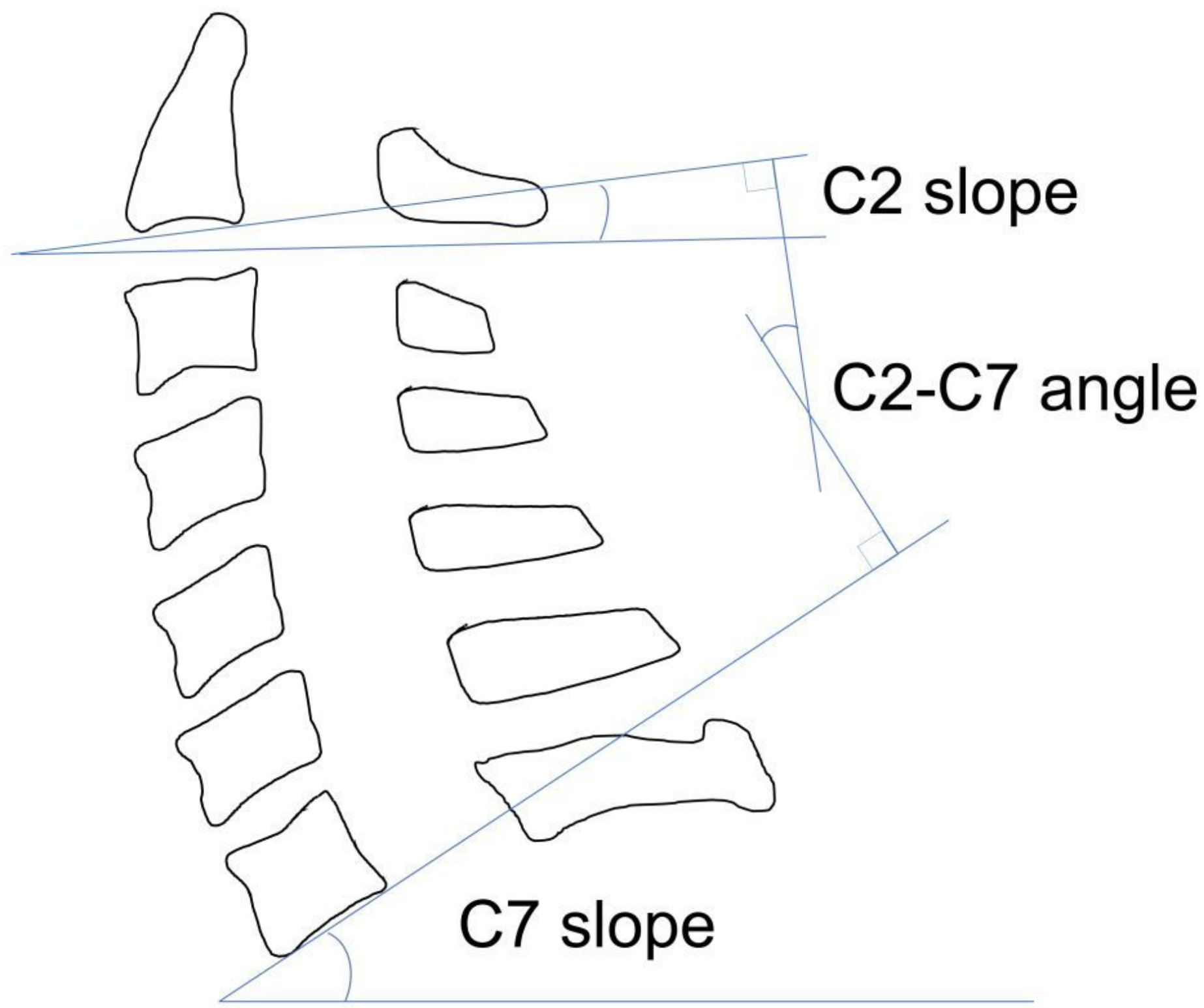

Figure 1

The $\mathrm{C} 2$ slope is the angle between the $\mathrm{C} 2$ lower endplate and the horizontal line, and the $\mathrm{C} 7$ slope is the angle between the $\mathrm{C} 7$ lower endplate and the horizontal line. The $\mathrm{C} 2-\mathrm{C} 7$ angle is the angle between C2slope and C7slope. 


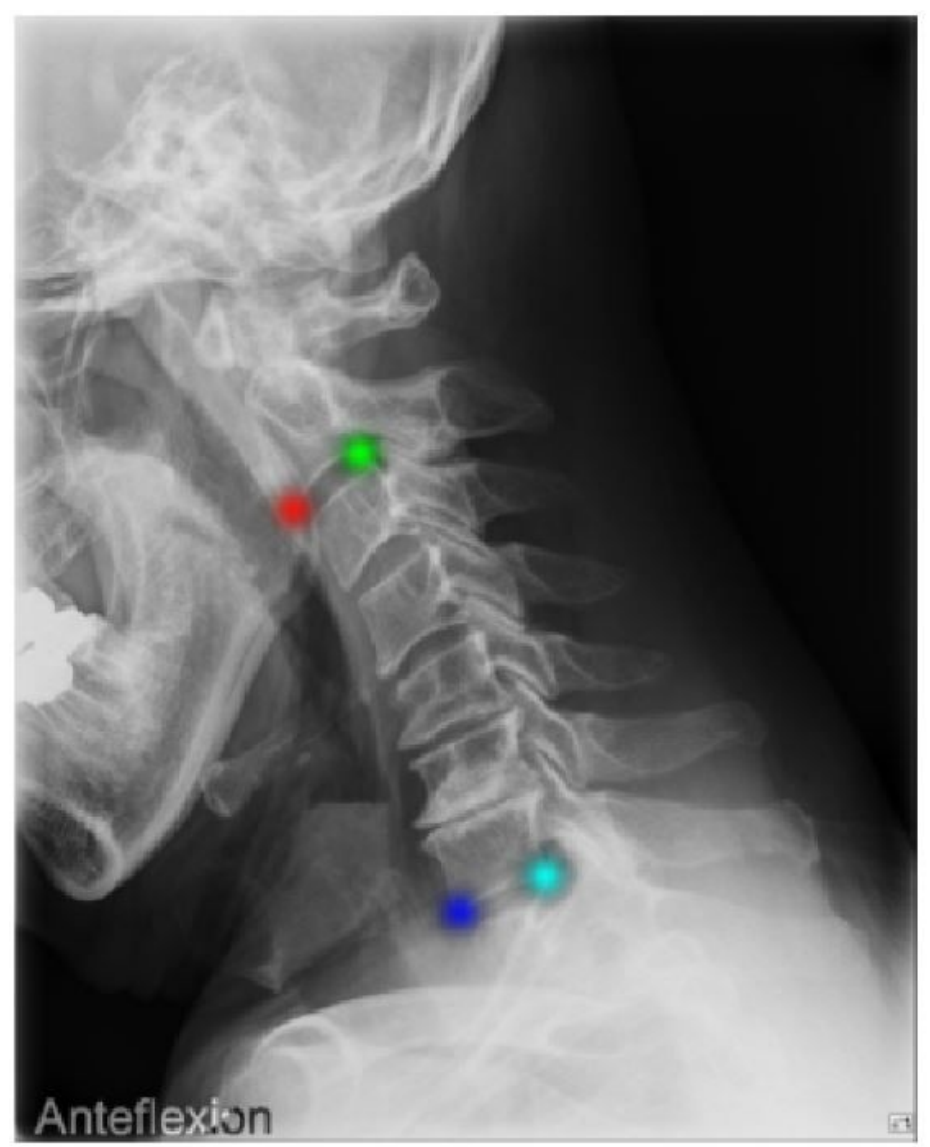

(a) Heatmaps

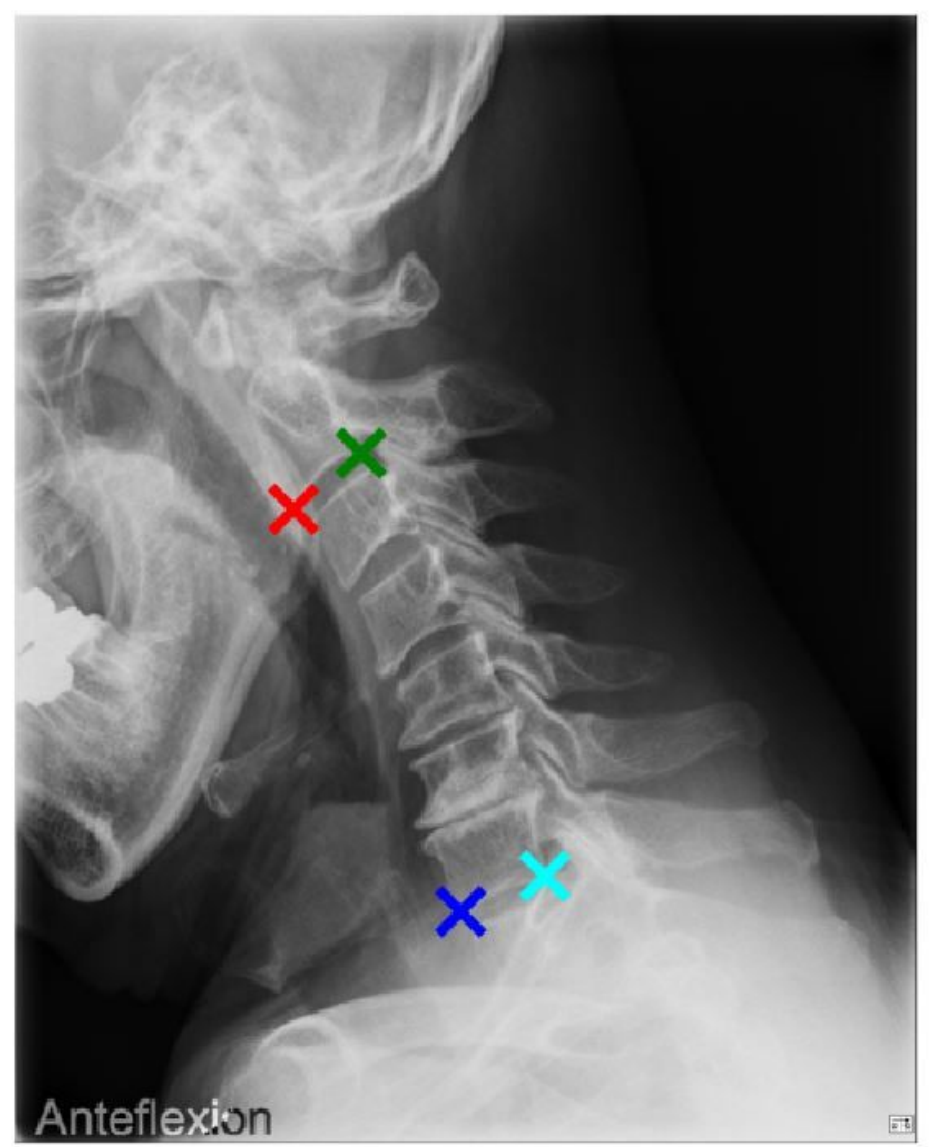

(b) Landmark point

Figure 2

Anatomic landmark localization is done by extracting the coordinates with the maximum value from the heat map (a) of each landmark (b) output by the convolutional neural networks. 

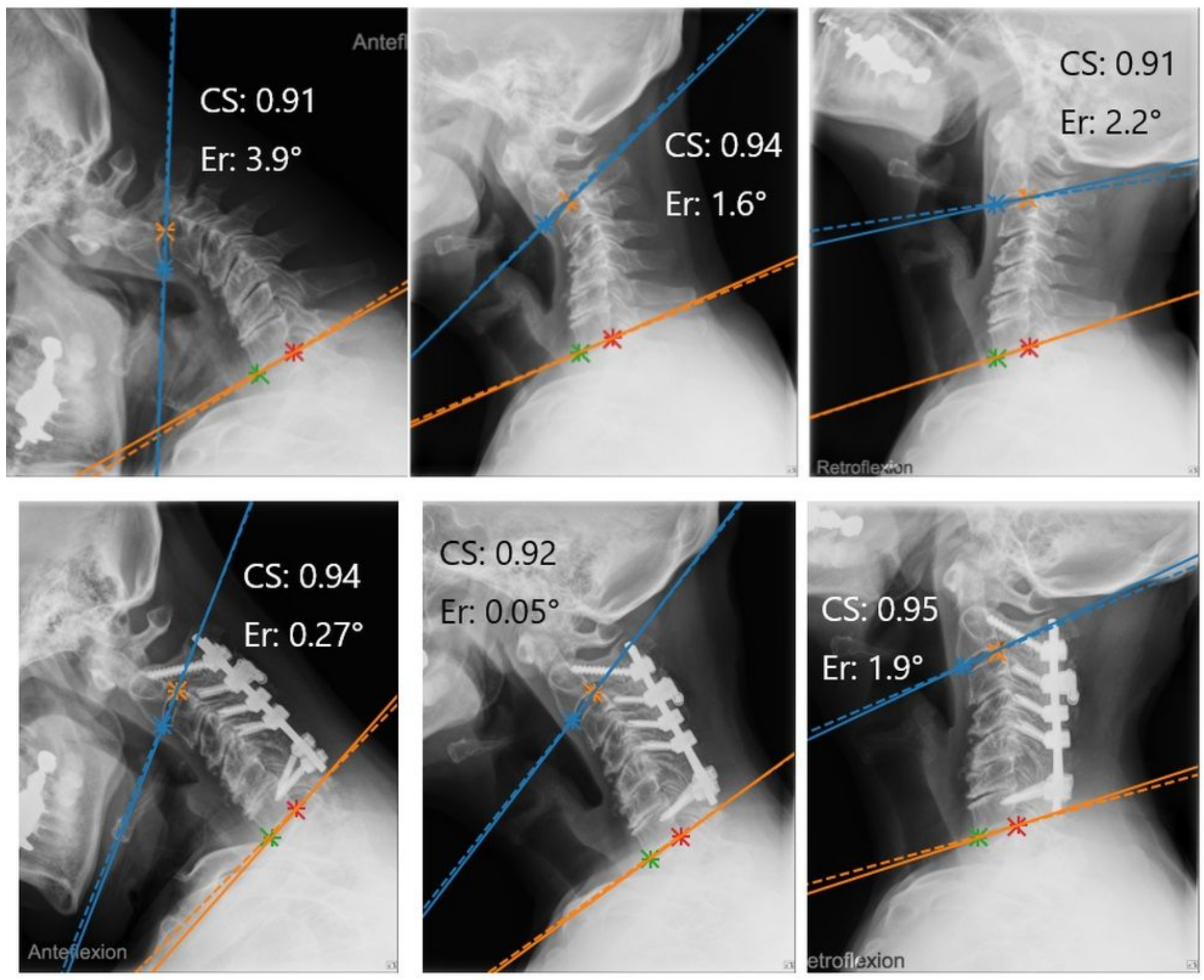

\section{Figure 3}

Preoperative (upper) and postoperative (lower) x-rays of a 54-year-old man. The solid lines represent the ground truth, and the dashed lines represent the measurement obtained by artificial intelligence. CS, confidence score; Er, error. 

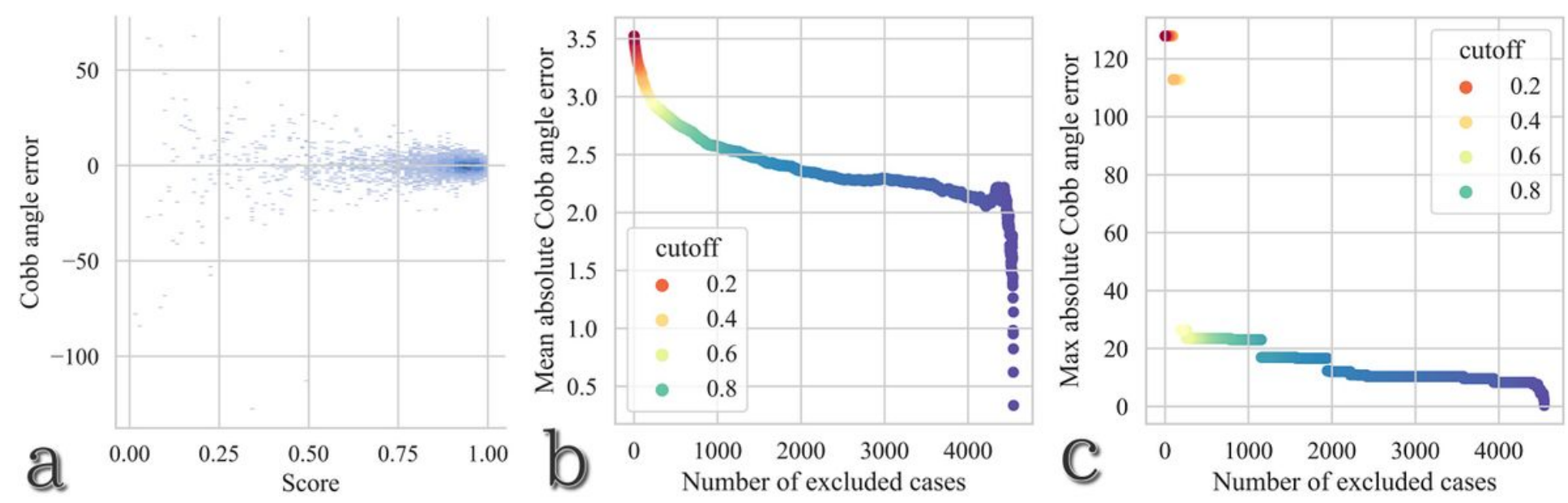

Figure 4

(a) Scatter plot showing the relationship between the confidence score and the error at the $\mathrm{C} 2-\mathrm{C} 7$ angle. The smaller the confidence score, the larger the error. (b) Relationship between number of excluded x-rays and mean absolute error at the $\mathrm{C} 2-\mathrm{C} 7$ angle when the cutoff value of the confidence score is changed. Increasing the threshold reduces the error but increases the number of $x$-rays to be excluded. (c) Relationship between the number of excluded x-rays and maximum absolute error at the $\mathrm{C} 2-\mathrm{C} 7$ angle when the cutoff value of the confidence score is changed.

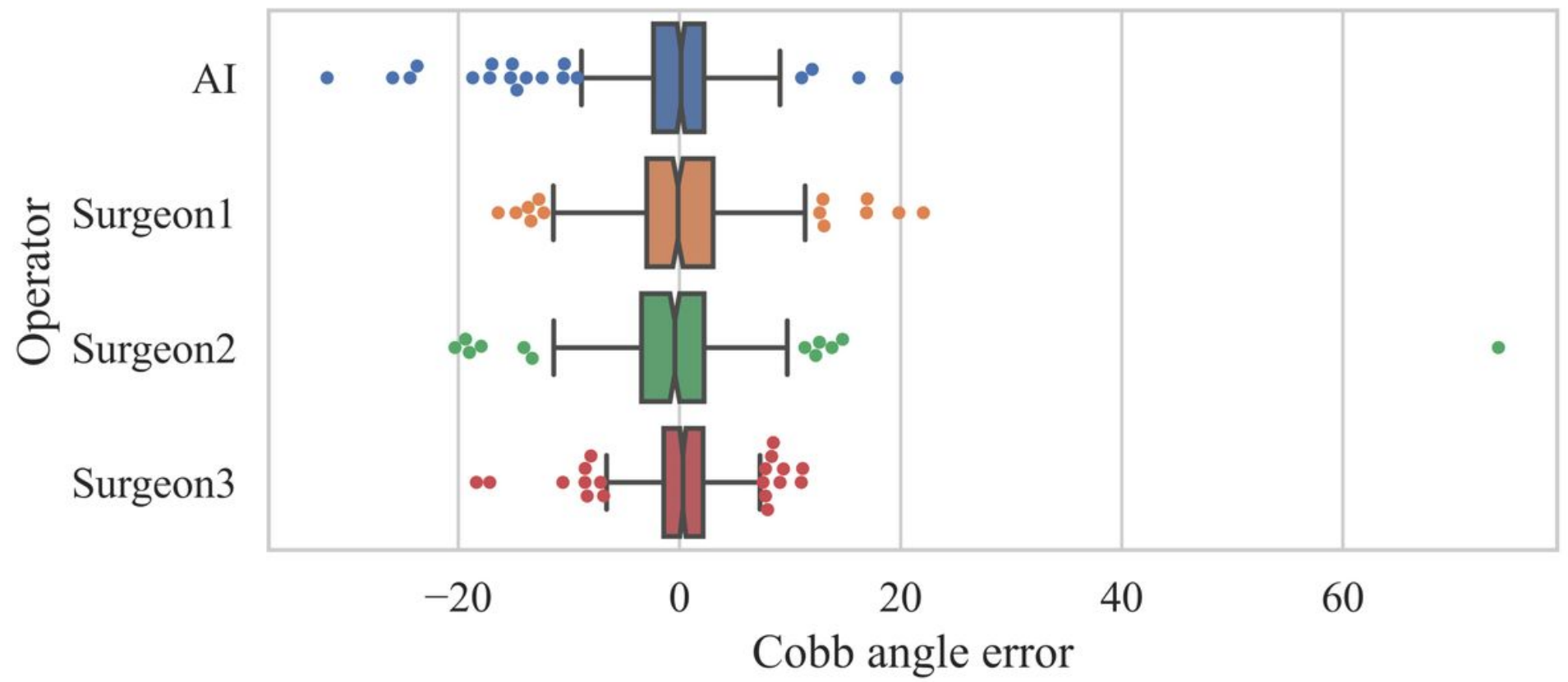

\section{Figure 5}

Box plot of the errors at the $\mathrm{C} 2-\mathrm{C} 7$ angle for 168 randomly selected participants with a total of $416 \mathrm{x}$ rays. The top of the box represents the 75 th percentile, the bottom of the box represents the 25th percentile, and the line in the middle represents the 50th percentile. The whiskers represent the highest 
and lowest values that are not outliers or extreme values. Dots beyond the whiskers represent outliers and extreme values. Al, artificial intelligence.

\section{Supplementary Files}

This is a list of supplementary files associated with this preprint. Click to download.

- demo.zip 